\title{
AN EXAMPLE OF KAC ALGEBRA ACTIONS ON VON NEUMANN ALGEBRAS
}

\author{
TAKEHIKO YAMANOUCHI
}

(Communicated by Palle E. T. Jorgensen)

\begin{abstract}
By using Majid's bicrossproduct Kac algebra, a nontrivial example of an action of a Kac algebra on a von Neumann algebra is given. It is shown that the actions constructed are ergodic. The crossed products by the actions are examined.
\end{abstract}

\section{INTRODUCTION}

The theory of Kac algebras defined in [ES] has been studied as a right framework for formulating Pontryagin-Tannaka-Krein-Tatsuuma duality for locally compact groups. By nature, the theory contains algebras $L^{\infty}(G)$ and $\mathscr{R}(G)=$ the group von Neumann algebra of $G$, as its typical examples, where $G$ is any locally compact group. Meanwhile, the concept of a coaction of a locally compact group $G$ was proven to be essential for duality for crossed products of von Neumann algebras, and it was noted that the Kac algebras $L^{\infty}(G)$ and $\mathscr{R}(G)$ play vital parts in the duality (see [NT] for the details). Motivated by this, Enock introduced in [E] the notion of an action of a Kac algebra on a von Neumann algebra. Group actions and group coactions supply typical examples of this notion. Every coproduct of a Kac algebra may also be considered as an action of the Kac algebra on itself; however, to the best of the author's knowledge, those are the only examples to date. Thus they are, so to speak, trivial examples in that one would not need to introduce such a concept of a Kac algebra action in the first place as long as they were the only examples. In this sense, the theory is short of examples. In order to find a "nontrivial" example, one would first need to look for a noncommutative and noncocommutative Kac algebra that would act on a von Neumann algebra. From this point of view, the recent work of Majid deserves to be noted. In [M] he studied the notion of a matched pair of locally compact groups and their actions and provided a lot of examples. He also proved there that the crossed products associated with a modular matched pair are equipped with a Kac algebraic structure; he called them the bicrossproduct Kac algebras. These Kac algebras are noncommutative and noncocommutative except in the trivial case. The purpose of this paper

Received by the editors October 28, 1991 and, in revised form, February 14, 1992.

1991 Mathematics Subject Classification. Primary 46L10, 46L55; Secondary 22D25.

Key words and phrases. Bicrossproducts, matched pairs, Kac algebras, actions of Kac algebras. 
is to construct a "nontrivial" example of an action of a Kac algebra on a von Neumann algebra by making good use of the bicrossproduct Kac algebras.

The organization of the paper is as follows. In $\S 1$ we first briefly review the definition of a Kac algebra and the notion of a Kac algebra action. We then recall the concept of a matched pair and the definition of the bicrossproduct Kac algebras associated with it. Section 2 is devoted to the construction of a nontrivial example of a Kac algebra action by using the bicrossproduct Kac algebras. It is shown that the actions constructed are ergodic. We also examine the crossed products by the actions.

\section{Preliminaries}

In this section, we first review the definition of a Kac algebra [ES] (see also [S1]) and the notion of an action of a Kac algebra on a von Neumann algebra [E]. Second, we recall the concept of a matched pair of locally compact groups and their actions. We also recall the definition of the bicrossproduct algebra associated with a matched pair.

A Kac algebra $\mathbf{K}$ is a quadruplet $(\mathscr{M}, \Gamma, \kappa, \varphi)$ in which

(Ki) $(\mathscr{M}, \Gamma, \kappa)$ is an involutive Hopf-von Neumann algebra [ES, Definition 1.2.1];

(Kii) $\varphi$ is a faithful, normal, semifinite weight on $\mathscr{M}$;

(Kiii) $\left(l_{\mathscr{M}} \otimes \varphi\right)(\Gamma(x))=\varphi(x) \cdot 1$ for all $x \in \mathscr{M}_{+}$;

(Kiv) $\left(l_{\mathscr{M}} \otimes \varphi\right)\left(\left(1 \otimes y^{*}\right) \Gamma(x)\right)=\kappa\left(\left(l_{\mathscr{M}} \otimes \varphi\right)\left(\Gamma\left(y^{*}\right)(1 \otimes x)\right)\right)$ for all $x, y \in N_{\varphi}$;

(Kv) $\sigma_{t}^{\varphi} \circ \kappa=\kappa \circ \sigma_{-t}^{\varphi}$ for all $t \in \mathbf{R}$.

Here $N_{\varphi}=\left\{x \in \mathscr{M}: \varphi\left(x^{*} x\right)<\infty\right\}$ and $\sigma^{\varphi}$ is the modular automorphism of $\varphi$. We will always think of $\mathscr{M}$ as represented in a standard form on the Hilbert space $\mathscr{H}_{\varphi}$ associated with $\varphi$. The $*$-isomorphism $\Gamma$ is called the coproduct (or the comultiplication) of $\mathbf{K}$. Given a Kac algebra $\mathbf{K}=(\mathscr{M}, \Gamma, \kappa, \varphi)$, there canonically exists another Kac algebra $\widehat{\mathbf{K}}=(\widehat{\mathscr{M}}, \widehat{\Gamma}, \hat{\kappa}, \hat{\varphi})$ called the dual Kac algebra of $\mathbf{K}$ [ES]. The pair $\left\{\widehat{\mathscr{M}}, \mathscr{H}_{\varphi}\right\}$ is again a standard representation.

To any locally compact group $G$, one can associate two canonical Kac algebras. One is the commutative Kac algebra $\operatorname{KA}(G)=\left(L^{\infty}(G), \Gamma_{G}, j_{G}, \tau_{G}\right)$ in which

$$
\begin{gathered}
\Gamma_{G}(f)(s, t)=f(s t), \quad j_{G}(f)(s)=f\left(s^{-1}\right), \\
\tau_{G}(f)=\int f(s) d s \quad\left(f \in L^{\infty}(G), s, t \in G\right),
\end{gathered}
$$

where $d s$ is a left Haar measure of $G$ and $L^{\infty}(G)$ is the algebra of all (equivalence classes of ) essentially bounded measurable functions on $G$ with respect to the left Haar measure. The other is the system $\operatorname{KS}(G)=\left(\mathscr{R}(G), \delta_{G}, \kappa_{G}, \varphi_{G}\right)$, where $\mathscr{R}(G)$ is the group von Neumann algebra of $G$. The morphisms $\delta_{G}$ and $\kappa_{G}$ are characterized by the identities

$$
\delta_{G}(\lambda(s))=\lambda(s) \otimes \lambda(s), \quad \kappa_{G}(\lambda(s))=\lambda\left(s^{-1}\right) \quad(s \in G) .
$$

Here $\lambda$ denotes the left regular representation of $G$. The weight $\varphi_{G}$ is the so-called Plancherel weight of $G$ that is derived from the left Hilbert algebra $\mathscr{K}(G)$, the set of all continuous functions on $G$ with compact support, with the usual convolution as its product. The $\operatorname{Kac}$ algebra $\operatorname{KS}(G)$ is cocommutative or symmetric. $\mathbf{K A}(G)$ and $\mathbf{K S}(G)$ are dual to each other. 
An action of a Kac algebra $\mathbf{K}=(\mathscr{M}, \Gamma, \kappa, \varphi)$ on a von Neumann algebra $\mathscr{P}$ is a $*$-isomorphism $\delta$ of $\mathscr{P}$ into $\mathscr{P} \bar{\otimes} \mathscr{M}$ satisfying $\delta(1)=1$ and

$$
\left(\delta \otimes l_{\mathscr{M}}\right) \circ \delta=\left(l_{\mathscr{P}} \otimes \Gamma\right) \circ \delta .
$$

In [E] the above action is referred to as a right action of $\mathbf{K}$ on $\mathscr{P}$, but, in this paper, we simply call it an action. Note that every coproduct of a Kac algebra $\mathbf{K}$ is an action of $\mathbf{K}$ on itself. The ordinary notion of an action of a locally compact group $G$ on a von Neumann algebra $\mathscr{P}$ (i.e., an automorphic representation of $G$ on $\mathscr{P}$ ) is, as shown in [NT, E], equivalent to an action of the Kac algebra $\mathbf{K A}(G)^{\sigma}$ on $\mathscr{P}$. (See [S2] for the definition of $\mathbf{K}^{\sigma}$.) In turn, an action of the cocommutative $\mathrm{Kac}$ algebra $\mathbf{K S}(G)$ on $\mathscr{P}$ is precisely the same as a coaction of $G$ on $\mathscr{P}$ in the sense of [NT]. Given an action $\delta$ of a Kac algebra $\mathbf{K}=(\mathscr{M}, \Gamma, \kappa, \varphi)$ on a von Neumann algebra $\mathscr{P}$, the crossed product of $\mathscr{P}$ by the action is defined in [E] to be the von Neumann algebra $\delta(\mathscr{P}) \vee \mathbf{C} \otimes \widehat{\mathscr{M}^{\prime}}$. We denote it by $\mathscr{P} \times_{\delta} \mathbf{K}$, or by $\mathscr{P} \times_{\delta} \mathscr{M}$ if there is no danger of confusion.

For the concept of a matched pair, we consider two locally compact groups $G_{1}$ and $G_{2}$ with their left Haar measures $\mu_{1}$ and $\mu_{2}$, respectively. We assume that $G_{1}$ acts on and is at the same time acted on by the set $G_{2}$ continuously and nonsingularly. By nonsingularity of a group action, we mean that the action preserves the null sets with respect to the measure in question. We denote by $\alpha$ (resp. $\beta$ ) the action of $G_{1}$ (resp. $G_{2}$ ). We shall keep using the letters $\alpha$, $\beta$ for the induced actions of $G_{1}$ and $G_{2}$ on algebras $L^{\infty}\left(G_{2}\right)$ and $L^{\infty}\left(G_{1}\right)$, respectively. Namely, we have

$$
\alpha_{g}(k)(s)=k\left(\alpha_{g^{-1}}(s)\right), \quad \beta_{s}(f)(g)=f\left(\beta_{s^{-1}}(g)\right),
$$

where $k \in L^{\infty}\left(G_{2}\right), f \in L^{\infty}\left(G_{1}\right), g \in G_{1}$, and $s \in G_{2}$. By assumption, it makes sense to consider the Radon-Nikodým derivatives

$$
\chi(g, s)=\frac{d \mu_{2} \circ \alpha_{g}}{d \mu_{2}}(s), \quad \Psi(s, g)=\frac{d \mu_{1} \circ \beta_{s}}{d \mu_{1}}(g) \quad\left(g \in G_{1}, s \in G_{2}\right) .
$$

The functions $\chi$ and $\Psi$ are cocycles on $G_{1} \times G_{2}$ and are assumed to be jointly continuous. We further assume that the actions $\alpha$ and $\beta$ satisfy the compatibility conditions:

$$
\begin{array}{ll}
\alpha_{g}(e)=e, & \beta_{s}(e)=e, \\
\alpha_{g}(s t)=\alpha_{\beta_{t}(g)}(s) \alpha_{g}(t), & \beta_{s}(g h)=\beta_{\alpha_{h}(s)}(g) \beta_{s}(h),
\end{array}
$$

where $g, h \in G_{1}$ and $s, t \in G_{2}$. In this case, we say that the system $\left(G_{1}, G_{2}\right.$, $\alpha, \beta)$ is a matched pair. We refer the reader to Lemma 2.2 of [M] for the properties that $\chi$ and $\Psi$ enjoy in case of $\left(G_{1}, G_{2}, \alpha, \beta\right)$ being a matched pair. A matched pair $\left(G_{1}, G_{2}, \alpha, \beta\right)$ is said to be modular [M] if

$$
\frac{\chi(g, s)}{\chi(g, e)}=\frac{\Psi(s, g)}{\Psi(s, e)}=1, \quad \frac{\delta_{2}\left(\alpha_{g}(s)\right)}{\delta_{2}(s)}=\frac{\delta_{1}\left(\beta_{s}(g)\right)}{\delta_{1}(g)}
$$

for all $g \in G_{1}$ and $s \in G_{2}$, where $\delta_{i}(i=1,2)$ indicates the modular functions of $G_{i}$. In [M] Majid gave abundunt examples of (modular) matched pairs of Lie groups and their actions. He also showed that if $\left(G_{1}, G_{2}, \alpha, \beta\right)$ is a matched pair, the crossed products $L^{\infty}\left(G_{2}\right) \times_{\alpha} G_{1}$ and $L^{\infty}\left(G_{1}\right) \times_{\beta} G_{2}$ can be equipped with a structure of an involutive Hopf-von Neumann algebra (see [ES] for the 
definition of an involutive Hopf-von Neumann algebra). He called the crossed products the bicrossproduct Hopf-von Neumann algebras. In particular, with the additional condition that the matched pair is modular, the crossed products become Kac algebras (see [ES]), dual to each other. He then called the algebras the bicrossproduct Kac algebras. We remark that these involutive Hopf-von Neumann algebras are not commutative or cocommutative except in the trivial case.

\section{Construction of AN EXAMPLE of A KaC ALgebra ACtion}

In this section, we construct an example of an action of a Kac algebra, which is not necessarily commutative or cocommutative, on a von Neumann algebra. The construction will be done by making use of the bicrossproduct Kac algebra associated with a modular matched pair.

Throughout this section, we shall fix a modular matched pair $\left(G_{1}, G_{2}, \alpha, \beta\right)$. We denote by $\mathbf{K}=(\mathscr{M}, \Gamma, \kappa, \varphi)$ the associated bicrossproduct Kac algebra, where $\mathscr{M}=L^{\infty}\left(G_{2}\right) \times_{\alpha} G_{1}$. With respect to our purpose, we are most interested in the coproduct $\Gamma$ of $\mathbf{K}$. So let us briefly recall how the morphism $\Gamma$ was defined in [M]. Let $\mathscr{H}_{i}(i=1,2)$ denote the Hilbert space $L^{2}\left(G_{i}\right)$. Then we set $\mathscr{H}=\mathscr{H}_{1} \otimes \mathscr{H}_{2}$. We define a unitary operator $W$ on $\mathscr{H} \otimes \mathscr{H}$, which is regarded as the set of $L^{2}$-functions on $G_{1} \times G_{2} \times G_{1} \times G_{2}$, by

$$
\{W \xi\}(g, s, h, t)=\xi\left(\beta_{t}(h)^{-1} g, s, h, \alpha_{\beta_{t}(h)^{-1} g}(s) t\right) \quad(\xi \in \mathscr{H} \otimes \mathscr{H}) .
$$

The inverse $W^{*}$ of this operator is given by

$$
\left\{W^{*} \xi\right\}(g, s, h, t)=\xi\left(\beta_{\alpha_{g}(s)^{-1} t}(h) g, s, h, \alpha_{g}(s)^{-1} t\right) .
$$

(See the proof of Theorem 2.6 of $[\mathrm{M}]$ for $W$.) The coproduct $\Gamma$ of $\mathbf{K}$ is defined by the identity

$$
\Gamma(x)=W(1 \otimes x) W^{*} \quad(x \in \mathscr{M}) .
$$

The next lemma illustrates what $\Gamma$ does to typical elements $\alpha(k) \quad\left(k \in L^{\infty}\left(G_{2}\right)\right)$ and $\lambda_{1}(p) \quad\left(p \in G_{1}\right)$ of the algebra $\mathscr{M}$.

Lemma 2.1. With the notation as above, we have

$$
\begin{gathered}
\{\Gamma(\alpha(k)) \xi\}(g, s, h, t)=k\left(\alpha_{g}(s) \alpha_{h}(t)\right) \xi(g, s, h, t), \\
\left\{\Gamma\left(\lambda_{1}(p) \otimes 1\right) \xi\right\}(g, s, h, t)=\xi\left(\beta_{\alpha_{h}(t)}\left(p^{-1}\right) g, s, p^{-1} h, t\right)
\end{gathered}
$$

where $\xi \in \mathscr{H} \otimes \mathscr{H}$.

A proof of the assertion is implicit in that of Theorem 2.6 of $[\mathrm{M}]$, thus we omit the details here.

Now we would like to let the Kac algebra $\mathscr{M}=L^{\infty}\left(G_{2}\right) \times_{\alpha} G_{1}$ act on the abelian von Neumann algebra $L^{\infty}\left(G_{2}\right)$. To do this, we first introduce a unitary operator $V_{\alpha}$ on the Hilbert space $\mathscr{H}_{2} \otimes \mathscr{H}$, which is regarded as the set of all $L^{2}$-functions on $G_{2} \times G_{1} \times G_{2}$, by

$$
\left\{V_{\alpha} \zeta\right\}(r, g, s)=\chi(g, e)^{1 / 2} \zeta\left(r, g, r \alpha_{g}(s)\right) \quad\left(\zeta \in \mathscr{H}_{2} \otimes \mathscr{H}\right) .
$$

Once we note that $\chi(g, s)=\chi(g, e)$ by modularity, it is not difficult to show that the operator $V_{\alpha}$ is indeed unitary with the inverse $V_{\alpha}^{*}$ given as

$$
\left\{V_{\alpha}^{*} \zeta\right\}(r, g, s)=\chi(g, e)^{-1 / 2} \zeta\left(r, g, \alpha_{g^{-1}}\left(r^{-1} s\right)\right) .
$$


We then define a $*$-isomorphism $\delta_{\alpha}$ of $L^{\infty}\left(G_{2}\right)$ into $\mathscr{L}\left(H_{2} \otimes \mathscr{H}\right)$ by

$$
\delta_{\alpha}(k)=V_{\alpha}\left(1_{\mathscr{K}_{2}} \otimes 1_{\mathscr{H}_{1}} \otimes k\right) V_{\alpha}^{*} \quad\left(k \in L^{\infty}\left(G_{2}\right)\right) .
$$

Here $\mathscr{L}(\mathscr{K})$ stands for the algebra of all bounded operators on a Hilbert space $\mathscr{K}$. Our aim is to prove that this morphism $\delta_{\alpha}$ gives an action of $\mathscr{M}$ on $L^{\infty}\left(G_{2}\right)$.

Lemma 2.2. We have $\left\{\delta_{\alpha}(k) \zeta\right\}(r, g, s)=k\left(r \alpha_{g}(s)\right) \zeta(r, g, s)$ for any $k \in$ $L^{\infty}\left(G_{2}\right)$ and $\zeta \in \mathscr{H}_{2} \otimes \mathscr{H}$. The image of $L^{\infty}\left(G_{2}\right)$ under the morphism $\delta_{\alpha}$ is contained in $L^{\infty}\left(G_{2}\right) \bar{\otimes} \mathscr{M}$.

Proof. The first assertion is easily verified by a simple calculation, so we leave its verification to the reader. For the second assertion, we define, for each $r \in G_{2}$, the function $k_{r}$ on $G_{2}$ by $k_{r}(s)=k(r s) \quad\left(s \in G_{2}\right)$. Then, with the notation introduced so far, we have that $k\left(r \alpha_{g}(s)\right)=k_{r}\left(\alpha_{g}(s)\right)=\alpha\left(k_{r}\right)(g, s)$. We note that the function $r \in G_{2} \mapsto \alpha\left(k_{r}\right)$ is an essentially bounded $\mathscr{M}$-valued function on $G_{2}$, so it belongs to $L^{\infty}\left(G_{2}, \mathscr{M}\right)=L^{\infty}\left(G_{2}\right) \bar{\otimes} \mathscr{M}$. Hence, by the first assertion, the operator $\delta_{\alpha}(k)$ lies in the von Neumann algebra $L^{\infty}\left(G_{2}\right) \bar{\otimes} \mathscr{M}$. Q.E.D.

Theorem 2.3. The *-isomorphism $\delta_{\alpha}$ defined above is an action of the Kac algebra $\mathscr{M}=L^{\infty}\left(G_{2}\right) \times_{\alpha} G_{1}$ on the von Neumann algebra $L^{\infty}\left(G_{2}\right)$.

Proof. For any $a \in L^{\infty}\left(G_{2}\right)$ and $x \in \mathscr{M}$, we have

$$
\begin{aligned}
\left(\delta_{\alpha} \otimes l_{\mathscr{K}}\right)(a \otimes x) & =\delta_{\alpha}(a) \otimes x=V_{\alpha}\left(1_{\mathscr{H}_{2}} \otimes 1_{\mathscr{K}_{1}} \otimes a\right) V_{\alpha}^{*} \otimes x \\
& =\left(V_{\alpha} \otimes 1_{\mathscr{H}}\right)\left(1_{\mathscr{H}_{2}} \otimes 1_{\mathscr{H}_{1}} \otimes a \otimes x\right)\left(V_{\alpha}^{*} \otimes 1_{\mathscr{H}}\right) .
\end{aligned}
$$

This shows that $\left(\delta_{\alpha} \otimes l_{\mathscr{M}}\right)(X)=\operatorname{Ad}\left(V_{\alpha} \otimes 1_{\mathscr{H}}\right)\left(1_{\mathscr{H}_{2}} \otimes 1_{\mathscr{H}_{1}} \otimes X\right)$ for any $X \in$ $L^{\infty}\left(G_{2}\right) \bar{\otimes}$. Hence, for any $k \in L^{\infty}\left(G_{2}\right)$ and $\xi \in \mathscr{H}_{2} \otimes \mathscr{H} \otimes \mathscr{H}$, which is considered as the set of all $L^{2}$-functions on $G_{2} \times G_{1} \times G_{2} \times G_{1} \times G_{2}$, we have

$$
\begin{aligned}
\left\{\left(\delta_{\alpha}\right.\right. & \left.\left.\otimes l_{\mathscr{M}}\right) \circ \delta_{\alpha}(k) \xi\right\}(r, g, s, h, t) \\
& =\left\{\left(V_{\alpha} \otimes 1_{\mathscr{K}}\right)\left(1_{\mathscr{K}_{2}} \otimes 1_{\mathscr{H}_{1}} \otimes \delta_{\alpha}(k)\right)\left(V_{\alpha}^{*} \otimes 1_{\mathscr{X}}\right) \xi\right\}(r, g, s, h, t) \\
& =\chi(g, e)^{1 / 2}\left\{\left(1_{\mathscr{K}_{2}} \otimes 1_{\mathscr{H}_{1}} \otimes \delta_{\alpha}(k)\right)\left(V_{\alpha}^{*} \otimes 1_{\mathscr{L}}\right) \xi\right\}\left(r, g, r \alpha_{g}(s), h, t\right) \\
& =\chi(g, e)^{1 / 2} k\left(r \alpha_{g}(s) \alpha_{h}(t)\right)\left\{\left(V_{\alpha}^{*} \otimes 1_{\mathscr{H}}\right) \xi\right\}\left(r, g, r \alpha_{g}(s), h, t\right) \\
& =k\left(r \alpha_{g}(s) \alpha_{h}(t)\right) \xi(r, g, s, h, t) .
\end{aligned}
$$

In the meantime, for any $a$ and $x$ as above, we have

$$
\begin{aligned}
\left(l_{L^{\infty}\left(G_{2}\right)} \otimes \Gamma\right)(a \otimes x) & =a \otimes \Gamma(x)=a \otimes W\left(1_{\mathscr{H}} \otimes x\right) W^{*} \\
& =\left(1_{L^{\infty}\left(G_{2}\right)} \otimes W\right)\left(a \otimes 1_{\mathscr{H}} \otimes x\right)\left(1_{L^{\infty}\left(G_{2}\right)} \otimes W^{*}\right) .
\end{aligned}
$$

It follows from this that $\left(l_{L^{\infty}\left(G_{2}\right)} \otimes \Gamma\right)(X)=\operatorname{Ad}\left(1_{L^{\infty}\left(G_{2}\right)} \otimes W\right)\left(X_{1,3}\right)$ for any $X \in L^{\infty}\left(G_{2}\right) \bar{\otimes}$. Here $X_{1,3}$ is the operator on $\mathscr{H}_{2} \otimes \mathscr{H} \otimes \mathscr{H}$ given by the equation $X_{1,3}=\left(\sigma \otimes 1_{\mathscr{L}}\right)(1 \otimes X)\left(\sigma \otimes 1_{\mathscr{H}}\right)$, where $\sigma$ in general denotes the unitary between tensor products $\mathscr{K}_{1} \otimes \mathscr{K}_{2}$ and $\mathscr{K}_{2} \otimes \mathscr{K}_{1}$ of Hilbert spaces $\mathscr{K}_{1}$, $\mathscr{K}_{2}$ defined by flipping vectors $\sigma\left(\eta_{1} \otimes \eta_{2}\right)=\eta_{2} \otimes \eta_{1}$. Thus, for $k$ and $\xi$ as 
before, we obtain

$$
\begin{aligned}
\left\{\left(l_{L^{\infty}\left(G_{2}\right)} \otimes \Gamma\right) \circ \delta_{\alpha}(k) \xi\right\}(r, g, s, h, t) \\
\quad=\left\{\left(1_{L^{\infty}\left(G_{2}\right)} \otimes W\right) \delta_{\alpha}(k)_{1,3}\left(1_{L^{\infty}\left(G_{2}\right)} \otimes W^{*}\right) \xi\right\}(r, g, s, h, t) \\
\quad=\left\{\delta_{\alpha}(k)_{1,3}\left(1_{L^{\infty}\left(G_{2}\right)} \otimes W^{*}\right) \xi\right\}\left(r, \beta_{t}(h)^{-1} g, s, h, \alpha_{\beta_{t}(h)^{-1} g}(s) t\right) \\
\quad=k\left(r \alpha_{h}\left(\alpha_{\beta_{t}(h)^{-1} g}(s) t\right)\right)\left\{\left(1_{L^{\infty}\left(G_{2}\right)} \otimes W^{*}\right) \xi\right\}\left(r, \beta_{t}(h)^{-1} g, s, h, \alpha_{\beta_{t}(h)^{-1} g}(s) t\right) \\
\quad=k\left(r \alpha_{h}\left(\alpha_{\beta_{t}(h)^{-1} g}(s) t\right)\right) \xi(r, g, s, h, t) .
\end{aligned}
$$

Now, from the compatibility condition (1.1), it follows that

$$
\alpha_{h}\left(\alpha_{\beta_{t}(h)^{-1} g}(s) t\right)=\alpha_{\beta_{t}(h)}\left(\alpha_{\beta_{t}(h)^{-1} g}(s)\right) \alpha_{h}(t)=\alpha_{g}(s) \alpha_{h}(t) .
$$

Consequently, we conclude that

$$
\left(\delta_{\alpha} \otimes l_{\mathscr{M}}\right) \circ \delta_{\alpha}(k)=\left(l_{L^{\infty}\left(G_{2}\right)} \otimes \Gamma\right) \circ \delta_{\alpha}(k)
$$

for any $k \in L^{\infty}\left(G_{2}\right)$. Therefore, the $*$-isomorphism $\delta_{\alpha}$ is an action of the Kac algebra $\mathscr{M}$ on $L^{\infty}\left(G_{2}\right)$. Q.E.D.

Remark 2.4. By symmetry, the bicrossproduct Kac algebra $L^{\infty}\left(G_{1}\right) \times_{\beta} G_{2}$, which is dual to $L^{\infty}\left(G_{2}\right) \times_{\alpha} G_{1}$, acts on the von Neumann algebra $L^{\infty}\left(G_{1}\right)$. The action $\delta_{\beta}$ is given by

$$
\delta_{\beta}(f)=V_{\beta}\left(1_{\mathscr{H}_{1}} \otimes f \otimes 1_{\mathscr{L}_{2}}\right) V_{\beta}^{*} \quad\left(f \in L^{\infty}\left(G_{1}\right)\right),
$$

where $V_{\beta}$ is a unitary on the Hilbert space $\mathscr{H}_{1} \otimes \mathscr{H}$, which is regarded as the set of all $L^{2}$-functions on $G_{1} \times G_{1} \times G_{2}$, defined by

$$
\left\{V_{\beta} \eta\right\}(g, h, s)=\Psi(s, e)^{1 / 2} \eta\left(g, g \beta_{s}(h), s\right) \quad\left(\eta \in \mathscr{H}_{1} \otimes \mathscr{H}\right) .
$$

The verification is left to the reader.

In what follows, we shall further investigate the action $\delta_{\alpha}$ of $\mathscr{M}$ on $L^{\infty}\left(G_{2}\right)$ to get much information on it; but before we begin to do so we need to introduce a notion of ergodicity of a Kac algebra action.

Definition 2.5. (1) Let $\delta$ be an action of a Kac algebra on a von Neumann algebra $\mathscr{P}$. Then the set

$$
\mathscr{P}^{\delta}=\{a \in \mathscr{P}: \delta(a)=a \otimes 1\}
$$

is clearly a von Neumann subalgebra of $\mathscr{P}$. It is called the fixed point algebra of the action $\delta$.

(2) Let $\delta$ be as above. We say that the action $\delta$ is ergodic if its fixed point algebra is trivial: $\mathscr{P}^{\delta}=\mathbf{C}$.

It is well known that if $\delta$ is an action of $\mathbf{K A}(G)^{\sigma}$ for some locally compact group $G$ then $\mathscr{P} \delta$ is the ordinary fixed point algebra. Thus ergodicity in our sense is consistent with the conventional notion of ergodicity of a group action.

Proposition 2.6. The action $\delta_{\alpha}$ in Theorem 2.3 is ergodic. Similarly, the action $\delta_{\beta}$ is ergodic.

Proof. We retain the notation introduced so far. Let $k \in L^{\infty}\left(G_{2}\right)$ be such that $\delta_{\alpha}(k)=k \otimes 1_{\mathscr{M}}$. We regard the von Neumann algebra $L^{\infty}\left(G_{2}\right) \bar{\otimes} \mathscr{M}$ as $L^{\infty}\left(G_{2}, \mathscr{M}\right)$, the set of all (equivalence classes of) essentially bounded $\mathscr{M}$ valued functions on $G_{2}$. Then, as we saw in the proof of Lemma 2.2, the 
operator $\delta_{\alpha}$ corresponds to the function $r \in G_{2} \mapsto \alpha\left(k_{r}\right)$, where $k_{r}(s)=k(r s)$. The element $k \otimes 1_{\mathscr{K}}$ in turn corresponds to the one $r \in G_{2} \mapsto k(r) \cdot 1_{\mathscr{M}}$. Hence the condition $\delta_{\alpha}(k)=k \otimes 1_{\mathscr{M}}$ is equivalent to the one that $\alpha\left(k_{r}\right)=k(r) \cdot 1_{\mathscr{M}}$ for $\mu_{2}$-a.e. $r \in G_{2}$. Fix an element $r \in G_{2}$ with $\alpha\left(k_{r}\right)=k(r) \cdot 1_{\mathscr{M}}$. Since

$$
\alpha\left(k_{r}\right)=k(r) \cdot 1_{\mathscr{M}}=\alpha\left(k(r) \cdot 1_{L^{\infty}\left(G_{2}\right)}\right),
$$

it follows from injectivity of $\alpha$ that $k_{r}=k(r) \cdot 1_{L^{\infty}\left(G_{2}\right)}$. Now it is easy to see that the function $k$ is constant a.e.; therefore, the action $\delta_{\alpha}$ is ergodic. Similarly, we can show ergodicity of $\delta_{\beta}$. Q.E.D.

Next we will look at the crossed product $L^{\infty}\left(G_{2}\right) \times_{\delta_{\alpha}} \mathscr{M}$ of $L^{\infty}\left(G_{2}\right)$ by the action $\delta_{\alpha}$ of $\mathscr{M}$. First we define a unitary representation $w$ of $G_{2}$ on $\mathscr{H}$ by

$$
\{w(t) \xi\}(g, s)=\delta_{2}\left(\alpha_{g}\left(t^{-1}\right)\right)^{-1 / 2} \Psi\left(t^{-1}, e\right)^{1 / 2} \xi\left(\beta_{t^{-1}}(g), s \alpha_{g}\left(t^{-1}\right)^{-1}\right),
$$

where $\xi \in \mathscr{H}$. Due to $(1.1)$ and modularity, $w(\cdot)$ is indeed a unitary representation.

Lemma 2.7. The action $\operatorname{Ad} w(t)$ of the group $G_{2}$ on $\mathscr{L}(\mathscr{H})$ leaves the algebra group $L^{\infty}\left(G_{1} \times G_{2}\right)$ globally invariant.

The proof is straightforward, so it is left to the reader.

Proposition 2.8. The crossed product $L^{\infty}\left(G_{2}\right) \times_{\delta_{\alpha}} \mathscr{M}$ is isomorphic to the von Neumann algebra generated by $L^{\infty}\left(G_{1} \times G_{2}\right)$ and $w\left(G_{2}\right)^{\prime \prime}$. Under the isomorphism, the center of the crossed product is the fixed point algebra $L^{\infty}\left(G_{1} \times G_{2}\right)^{\operatorname{Ad} w(\cdot)}$ of the action $\operatorname{Ad} w(\cdot)$ on $L^{\infty}\left(G_{1} \times G_{2}\right)$.

Proof. We denote the crossed product by $\mathscr{Q}$. By definition, $\mathscr{Q}$ is generated by $\delta_{\alpha}\left(L^{\infty}\left(G_{2}\right)\right)$ and $\mathbf{C} \otimes \widehat{\mathscr{M}^{\prime}}$. We note that the algebra $\widehat{\mathscr{M}^{\prime}}$ is engendered by $L^{\infty}\left(G_{1}\right) \otimes \mathbf{C}$ and $\left\{v(s) \otimes \rho_{2}(s): s \in G_{2}\right\}^{\prime \prime}$, where $\rho_{2}$ is the right regular representation of $G_{2}$ and $v(\cdot)$ is the unitary implementing the action $\beta$ :

$$
\{v(s) f\}(g)=\Psi\left(s^{-1}, g\right)^{1 / 2} f\left(\beta_{s^{-1}}(g)\right) \quad\left(f \in \mathscr{H}_{1}\right) .
$$

Hence, recalling the definition of the action $\delta_{\alpha}$, we deduce that the algebra $V_{\alpha}^{*} \mathscr{Q} V_{\alpha}$ is generated by $\mathbf{C} \otimes \mathbf{C} \otimes L^{\infty}\left(G_{2}\right), \quad V_{\alpha}^{*}\left(\mathbf{C} \otimes L^{\infty}\left(G_{1}\right) \otimes \mathbf{C}\right) V_{\alpha}$, and $V_{\alpha}^{*}\left\{1 \otimes v(s) \otimes \rho_{2}(s): s \in G_{2}\right\}^{\prime \prime} V_{\alpha}$. By the definition of $V_{\alpha}$, it is easy to see that

$$
V_{\alpha}^{*}(1 \otimes f \otimes 1) V_{\alpha}=1 \otimes f \otimes 1
$$

for all $f \in L^{\infty}\left(G_{1}\right)$. Thus $V_{\alpha}^{*}\left(\mathbf{C} \otimes L^{\infty}\left(G_{1}\right) \otimes \mathbf{C}\right) V_{\alpha}=\mathbf{C} \otimes L^{\infty}\left(G_{1}\right) \otimes \mathbf{C}$. Next we compute the following:

$$
\begin{aligned}
&\left\{V_{\alpha}^{*}\left(1 \otimes v(t) \otimes \rho_{2}(t)\right) V_{\alpha} \zeta\right\}(r, g, s) \\
&= \chi(g, e)^{-1 / 2}\left\{\left(1 \otimes v(t) \otimes \rho_{2}(t)\right) V_{\alpha} \zeta\right\}\left(r, g, \alpha_{g^{-1}}\left(r^{-1} s\right)\right) \\
&= \chi(g, e)^{-1 / 2} \delta_{2}(t)^{1 / 2} \Psi\left(t^{-1}, g\right)^{1 / 2}\left\{V_{\alpha} \zeta\right\}\left(r, \beta_{t^{-1}}(g), \alpha_{g^{-1}}\left(r^{-1} s\right) t\right) \\
&= \chi(g, e)^{-1 / 2} \delta_{2}(t)^{1 / 2} \Psi\left(t^{-1}, e\right)^{1 / 2} \chi\left(\beta_{t^{-1}}(g), e\right)^{1 / 2} \\
& \times \zeta\left(r, \beta_{t^{-1}}(g), r \alpha_{\beta_{t^{-1}}(g)}\left(\alpha_{g^{-1}}\left(r^{-1} s\right) t\right)\right),
\end{aligned}
$$

where $\zeta \in \mathscr{H}_{2} \otimes \mathscr{H}$. By the second identity of Lemma 2.2 in [M], we have

$$
\delta_{2}(t)^{1 / 2} \chi(g, e)^{-1 / 2} \chi\left(\beta_{t^{-1}}(g), e\right)^{1 / 2}=\delta_{2}\left(\alpha_{g}\left(t^{-1}\right)\right)^{-1 / 2} .
$$


Meanwhile, it results from (1.1) that

$$
\begin{aligned}
r \alpha_{\beta_{t^{-1}}(g)}\left(\alpha_{g^{-1}}\left(r^{-1} s\right) t\right) & =r \alpha_{\beta_{t}\left(\beta_{t^{-1}}(g)\right)}\left(\alpha_{g^{-1}}\left(r^{-1} s\right)\right) \alpha_{\beta_{t^{-1}}(g)}(t) \\
& =s \alpha_{\beta_{t^{-1}}(g)}(t)=s \alpha_{g}\left(t^{-1}\right)^{-1}
\end{aligned}
$$

The last step is due to the fact that $e=\alpha_{g}\left(t t^{-1}\right)=\alpha_{\beta_{t^{-1}}(g)}(t) \alpha_{g}\left(t^{-1}\right)$. Consequently, we obtain

$$
\begin{aligned}
\left\{V_{\alpha}^{*}\right. & \left.\left(1 \otimes v(t) \otimes \rho_{2}(t)\right) V_{\alpha} \zeta\right\}(r, g, s) \\
& =\delta_{2}\left(\alpha_{g}\left(t^{-1}\right)\right)^{-1 / 2} \Psi\left(t^{-1}, g\right)^{1 / 2} \zeta\left(r, \beta_{t^{-1}}(g), s \alpha_{g}\left(t^{-1}\right)^{-1}\right) \\
& =\delta_{2}\left(\alpha_{g}\left(t^{-1}\right)\right)^{-1 / 2} \Psi\left(t^{-1}, e\right)^{1 / 2} \zeta\left(r, \beta_{t^{-1}}(g), s \alpha_{g}\left(t^{-1}\right)^{-1}\right) \\
& =\{(1 \otimes w(t)) \zeta\}(r, g, s) .
\end{aligned}
$$

The second equality is guaranteed by the modularity $\Psi\left(t^{-1}, g\right)=\Psi\left(t^{-1}, e\right)$. From the above computation, it follows that

$$
\begin{aligned}
V_{\alpha}^{*} \mathscr{Q} V_{\alpha} & =\mathbf{C} \otimes \mathbf{C} \otimes L^{\infty}\left(G_{2}\right) \vee \mathbf{C} \otimes L^{\infty}\left(G_{1}\right) \otimes \mathbf{C} \vee \mathbf{C} \otimes w\left(G_{2}\right)^{\prime \prime} \\
& =\mathbf{C} \otimes L^{\infty}\left(G_{1} \otimes G_{2}\right) \vee w\left(G_{2}\right)^{\prime \prime} .
\end{aligned}
$$

Therefore the crossed product $\mathscr{Q}$ is isomorphic to $L^{\infty}\left(G_{1} \times G_{2}\right) \vee w\left(G_{2}\right)^{\prime \prime}$. It is now clear that the center of $\mathscr{Q}$ is isomorphic to $L^{\infty}\left(G_{1} \times G_{2}\right) \cap w\left(G_{2}\right)^{\prime}$, which coincides with the fixed point algebra $L^{\infty}\left(G_{1} \times G_{2}\right)^{\mathrm{Ad} w}$. Q.E.D.

Finally we close this section with a remark that a similar result holds true for the crossed product $L^{\infty}\left(G_{1}\right) \times_{\delta_{\beta}} \widehat{\mathscr{M}}$.

\section{ACKNOWLEDGMENT}

This work was partly done while the author stayed at Centre de Recherches Mathématiques, Université de Montréal, and Department of Mathematics, University of Toronto. The author would like to thank the staffs at these institutes for the hospitality extended to him. He also expresses his sincere gratitude to Professors David Handelman and George Elliott, who gave him an opportunity to visit these institutions and supported him during the period.

\section{REFERENCES}

[E] M. Enock, Produit croisé d'une algèbre de von Neumann par une algèbre de Kac, J. Funct. Anal. 26 (1977), 16-47.

[ES] M. Enock and J. M. Schwartz, Une dualité dans les algèbres de von Neumann, Bull. Soc. Math. France Supp. Mémoire 44 (1975), 1-144.

[M] S. Majid, Hopf-von Neumann algebra bicrossproducts, Kac algebra bicrossproducts and the classical Yang-Baxter equations, J. Funct. Anal. 95 (1991), 291-319.

[NT] Y. Nakagami and M. Takesaki, Duality for crossed products of von Neumann algebras, Lectures Notes in Math., vol. 731, Springer-Verlag, Berlin and New York, 1979.

[S1] J. M. Schwartz, Sur la structure des algèbres de Kac. I, J. Funct. Anal. 34 (1979), 370-406.

[S2] _ Sur la structure des algèbres de Kac. II, Proc. London Math. Soc. (3) 41 (1980), $465-480$. 
[T] M. Takesaki, Duality for crossed products and the structure of von Neumann algebras of type III, Acta Math. 131 (1973), 249-310.

[Y] T. Yamanouchi, The intrinsic group of Majid's bicrossproduct Kac algebra, Pacific J. Math. (to appear).

Department of Mathematics and Computer Science, University College of Swansea, Singleton Park, Swansea, SA2 8PP United Kingdom

Current address: Department of Mathematics, Faculty of Science, University of Hokkaido, Sapporo, 060 Japan

E-mail address: yamanou@euler.math.hokudai.ac.jp 